\title{
Correlation between magnetic spin structure and the three-dimensional geometry in chemically synthesized nanoscale magnetite rings
}

Eltschka, M.; Klaui, M.; Rudiger, U; Kasama, T; Gontard, Lionel Cervera; Dunin-Borkowski, Rafal E.; Luo, F; Heyderman, LJ; Jia, C-J; Sun, L-D

Total number of authors:

11

Published in:

Applied Physics Letters

Link to article, DOI:

$10.1063 / 1.2936989$

Publication date:

2008

Document Version

Publisher's PDF, also known as Version of record

Link back to DTU Orbit

Citation (APA):

Eltschka, M., Klaui, M., Rudiger, U., Kasama, T., Gontard, L. C., Dunin-Borkowski, R. E., Luo, F., Heyderman, LJ., Jia, C-J., Sun, L-D., \& Yan, C-H. (2008). Correlation between magnetic spin structure and the threedimensional geometry in chemically synthesized nanoscale magnetite rings. Applied Physics Letters, 92(22), 222508. https://doi.org/10.1063/1.2936989

\section{General rights}

Copyright and moral rights for the publications made accessible in the public portal are retained by the authors and/or other copyright owners and it is a condition of accessing publications that users recognise and abide by the legal requirements associated with these rights.

- Users may download and print one copy of any publication from the public portal for the purpose of private study or research.

- You may not further distribute the material or use it for any profit-making activity or commercial gain

- You may freely distribute the URL identifying the publication in the public portal 


\title{
Correlation between magnetic spin structure and the three-dimensional geometry in chemically synthesized nanoscale magnetite rings
}

\author{
M. Eltschka, ${ }^{1, a)}$ M. Kläuii, ${ }^{1, b)}$ U. Rüdiger, ${ }^{1}$ T. Kasama ${ }^{2}$ L. Cervera-Gontard ${ }^{3, a)}$ \\ R. E. Dunin-Borkowski, ${ }^{3, a)}$ F. Luo, ${ }^{4}$ L. J. Heyderman, ${ }^{4}$ C.-J. Jia, ${ }^{5}$ L.-D. Sun, ${ }^{5}$ and C.-H. Yan ${ }^{5}$ \\ ${ }^{1}$ Fachbereich Physik, Universität Konstanz, Universitätsstraße 10, 78457 Konstanz, Germany \\ ${ }^{2}$ Department of Materials Science and Metallurgy, University of Cambridge, Pembroke Street, Cambridge \\ CB2 3QZ, United Kingdom \\ ${ }^{3}$ Center for Electron Nanoscopy, Technical University of Denmark, DK-2800 Kongens Lyngby, Denmark \\ ${ }^{4}$ Laboratory for Micro- and Nanotechnology, Paul Scherrer Institut, 5232 Villigen PSI, Switzerland \\ ${ }^{5}$ Beijing National Laboratory for Molecular Sciences, State Key Laboratory of Rare Earth Materials \\ Chemistry and Applications and PKU-HKU Joint Laboratory in Rare Earth Materials and Bioinorganic \\ Chemistry, Peking University, Beijing 100871, People's Republic of China
}

(Received 4 February 2008; accepted 21 April 2008; published online 6 June 2008)

\begin{abstract}
The correlation between magnetic spin structure and geometry in nanoscale chemically synthesized $\mathrm{Fe}_{3} \mathrm{O}_{4}$ rings has been investigated by transmission electron microscopy. We find primarily the flux closure vortex states but in rings with thickness variations, an effective stray field occurs. Using tomography, we determine the complete three-dimensional geometries of thicker rings. A direct correlation between the geometry and the magnetization which points out of plane in the thickest parts of the ring yielding an intermediate magnetic state between the vortex state and the tube state is found. The interaction between exchange coupled rings leads to antiparallel vortex states and extended onion states. (c) 2008 American Institute of Physics. [DOI: 10.1063/1.2936989]
\end{abstract}

In recent years, ferromagnetic nanostructures have become the focus of increasing interest due to the trend toward miniaturization of physical systems down to dimensions where fundamental effects occur and due to possible applications. ${ }^{1,2}$ Such devices rely on large magnetoresistance (MR) effects, which in turn are achieved in materials with a high spin polarization. A particularly exciting class of materials are half metals, which exhibit a $100 \%$ spin polarization at the Fermi level. An example is magnetite $\left(\mathrm{Fe}_{3} \mathrm{O}_{4}\right)$, which has been extensively studied because it has a high Curie temperature $\left(T_{C}\right)$ of $858 \mathrm{~K}$ in addition to a high spin polarization. ${ }^{3}$ The magnetic properties of thin films of various thicknesses and bulk single crystal $\mathrm{Fe}_{3} \mathrm{O}_{4}$ have been investigated experimentally ${ }^{4,5}$ and large values of MR have been measured. 6,7

In general, the magnetic properties of ferromagnetic nanoscale elements are governed by the element geometry rather than by the intrinsic materials properties. ${ }^{8,9}$ A large effort has been spent studying magnetic states and magnetization reversal mechanisms in such nanostructures in order to find the geometry which provides the simplest, fastest, and most reproducible switching behavior of the magnetization. ${ }^{8-11}$ The magnetization configuration that has raised significant interest is the vortex state in circular elements due to a low influence of edge roughness and due to the zero stray field which makes it promising for applications in high-density storage. ${ }^{1}$ The highest stability of the vortex state is reached in high-symmetry ring elements where the only source of stray field, namely the highly energetic vortex core is removed. ${ }^{1,10,11}$ In the study of ring elements, different magnetic states (vortex, onion, and vortex core state) have been identified, depending on the geometry and applied external magnetic fields. ${ }^{12}$ Furthermore, the switching pro-

\footnotetext{
${ }^{\text {a) }}$ Also at the Department of Materials Science and Metallurgy, University of Cambridge, Pembroke Street, Cambridge CB2 3QZ, United Kingdom.

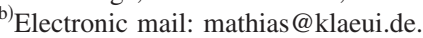

cesses have been investigated ${ }^{10,12,13}$ and the magnetic coupling via stray field interaction between adjacent rings has been measured. $^{14}$

So far these investigations have been carried out on rings fabricated using a top down approach, patterning ferromagnetic thin films with electron beam lithography. This technique has a number of drawbacks. The lift-off process used is not compatible with the high temperature deposition necessary for $\mathrm{Fe}_{3} \mathrm{O}_{4}$ and for large scale industrial applications, e-beam writing is slow and rather a self assembly technique is needed. Finally, the top down approach that we previously employed $^{15}$ only allowed for the fabrication of "thin" twodimensional rings where the thicknesses $t$ are much smaller than the widths $w(t \ll w)$ and the minimum ring size is limited by the electron beam lithography resolution. To overcome these problems, a bottom-up technique needs to be devised, which also allows for mass fabrication of "thick" three-dimensional rings $(t \geqslant w)$ which have not been explored so far. Such thick rings are of interest since they are more stable against thermal excitations and they allow the study of the transition to the "tube" geometry where the magnetization is expected to be aligned parallel to the tube axis rather than in the ring plane.

In this paper, we investigate the dependence of magnetization configurations in single crystal magnetite rings on their geometry. In contrast to the thin rings previously studied, the thickness of the rings fabricated by this bottom-up technique can be as large as the diameter allowing for the observation of the transition to a tubelike spin structure. By using noninvasive transmission electron microscopy (TEM) techniques, we investigate the geometry, the crystallography and the correlation to the magnetic properties of the magnetite rings. We observe different magnetization configurations depending on the detailed three-dimensional geometry of the rings. Additionally, we study the influence of the geometrical arrangement of multiple interacting rings. 


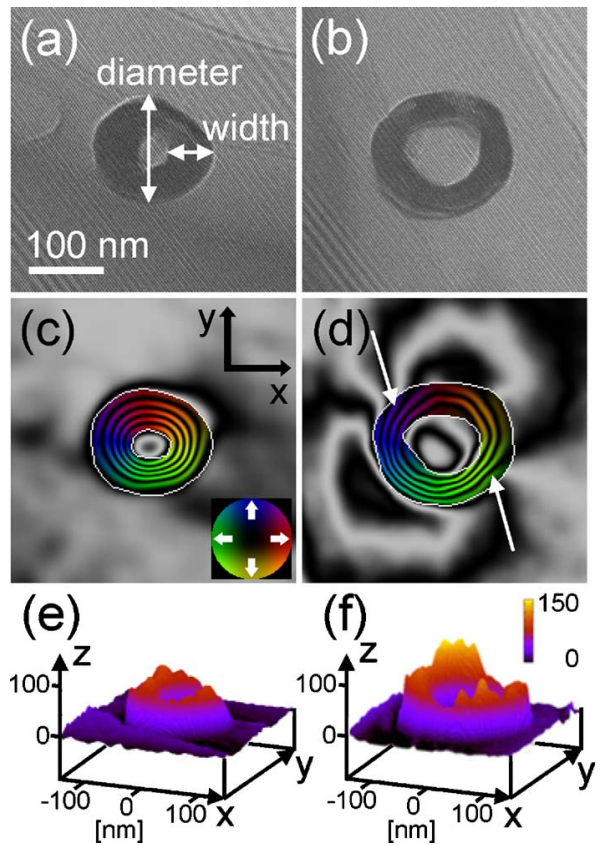

FIG. 1. (Color online) [(a) and (b)] Off-axis electron hologram of single magnetite rings. The phase changes due to the mean inner potential and magnetic potential can be seen from the interference fringes. [(c) and (d)] Direction of the magnetic induction at zero field after magnetization (the magnetization directions are given by the color wheel). In (c), the region around the ring shows a uniform contrast whereas in (d) a stray field is visible outside the ring. The position of the magnetic surface charges that are the origins of the stray field is indicated by arrows. [(e) and (f)] Thickness of the rings calculated by using the phase shift due to the mean inner potential. The thickness in (e) is more uniform than in (f), which varies between 40 and $120 \mathrm{~nm}$. The combination of (d) and (f) yields as the origins of the stray field the thickness variations of the ring.

Single crystal $\mathrm{Fe}_{3} \mathrm{O}_{4}$ nanorings, as shown in Figs. 1(a) and $1(\mathrm{~b})$, are fabricated via a reduction process with the corresponding $\alpha-\mathrm{Fe}_{2} \mathrm{O}_{3}$ products as precursors obtained by hydrothermal treatment of a mixture of $\mathrm{FeCl}_{3}, \mathrm{NH}_{4} \mathrm{H}_{2} \mathrm{PO}_{4}$, and $\mathrm{Na}_{2} \mathrm{SO}_{4}{ }^{15}$ The geometries of the rings can be varied in diameter, width, and thickness depending on the cooperative action of both phosphate and sulphate ions in the $\alpha-\mathrm{Fe}_{2} \mathrm{O}_{3}$ precursors which also allows for the fabrication of exchange coupled rings. ${ }^{15}$ We investigate ring diameters ranging from 150 to $250 \mathrm{~nm}$ and widths from 40 to $80 \mathrm{~nm}$. The thicknesses of the rings are between 70 and $150 \mathrm{~nm}$. The magnetite rings are deposited as isolated single rings or as chains of touching rings on TEM carbon grids which are suitable for taking electron holograms in a transmission electron microscope.

To investigate the crystal structure, we acquire diffraction patterns of isolated rings and clusters of rings in the transmission electron microscope. Comparing the results to computer-based simulations and reference values in literature indicates that the magnetite rings have a typical inverse spinel structure of magnetite with the [111] orientation of the fcc lattice along the $z$ direction (see Fig. 1 for coordinate system).

Since the ring structures are three-dimensional objects unlike the previously studied thin film rings, conventional two-dimensional imaging is not sufficient to determine the full geometrical structure. Therefore we employ high-angle annular dark-field (HAADF) electron tomography allowing three-dimensional atomic resolution. ${ }^{16} \mathrm{~A}$ series of projections at different tilting angles is taken and from its mass density Downloaded 21 Jun 2010 to 192.38 .67 .112 . Redistribution subject distribution a three-dimensional reconstruction is obtained.

To observe the magnetic properties of these structures, we use off-axis electron holography, which provides the necessary high spatial resolution $(<10 \mathrm{~nm}) .{ }^{17}$ The phase of the electron wave is shifted by electric and magnetic fields when crossing the sample. Using a biprism, the electron beam passing through the specimen interferes with a reference beam, and the interference patterns provide information about the magnitude and direction of the projected magnetic induction. This allows for the calculation of the magnetic induction map and the determination of the sample thickness from the mean inner potential. ${ }^{17}$

To understand the influence of the geometry on the magnetization, we first consider the magnetization of a single magnetite ring (ring 1: average diameter of $170 \mathrm{~nm}$, average width of $50 \mathrm{~nm}$ ), as shown in Fig. 1(a). We magnetize the ring by tilting the sample $\left(30^{\circ}\right)$ and applying a field with a resulting in-plane component of about 0.6 Tesla and an outof-plane component of about $1 \mathrm{~T}$ using the TEM objective lens. After relaxing the field, we take a hologram to extract the magnetic information shown in Fig. 1(c). We find that the ring is in the vortex state, which is the preferred state for storing information. When we reverse the field, the circulation direction reverses as the vortex reverses, which means that we can set the desired magnetic state. ${ }^{12}$ Outside the ring, virtually no stray field is visible, which is one of the virtues of the flux closure vortex state.

When measuring a similar single ring (ring 2: average diameter of $190 \mathrm{~nm}$, average width of $45 \mathrm{~nm}$ ), as shown in Fig. 1(b), we again observe a magnetic vortex state. However, in contrast to the first sample, stray field outside the ring is visible [Fig. 1(d)]. The small differences in ring diameter and width compared to Fig. 1(a) do not explain this observation. To examine thickness variations, we use $t / \lambda$ maps obtained using electron energy-loss spectroscopy, where $\lambda$ is the inelastic mean free path of electrons in the sample (170 nm for magnetite at an accelerating voltage of $300 \mathrm{kV}) .{ }^{18}$ As shown in Figs. 1(e) and 1(f), the thickness of the first ring has an average value of $70 \mathrm{~nm}$ and the thickness is nearly uniform. The second ring is not only thicker, but there are also large variations in thickness which ranges from 60 to $140 \mathrm{~nm}$. The comparison of the three-dimensional plot and the magnetic induction for the second ring [Figs. 1(d) and 1(f)] reveals that the thickness variations are the origin of the stray field. This is because the large flux from the thick parts of the ring cannot be contained inside the thin parts of the ring. For these thickness variations, this vortex state (with a small increase in the stray field energy) is energetically more favorable compared to a multidomain state.

The stray field interaction between rings with variable edge-to-edge spacing has also raised significant interest. ${ }^{14,19}$ Using off-axis electron holography, we look directly at exchange coupled rings which are touching as result of the fabrication process [Fig. 2(a)]. In this case, we find a vortex state in each ring with opposite circulation directions. This magnetization state is energetically favorable because the magnetic field is parallel in the contact region and does not require the formation of a domain wall that is necessary when the circulation directions are the same.

So far, all the rings observed have been in the vortex state. When a row of three or more rings is present, we can stabilize a different magnetic state by applying an external field with an in-plane component which is nearly parallel to to AIP license or copyright; see http://apl.aip.org/apl/copyright.jsp 

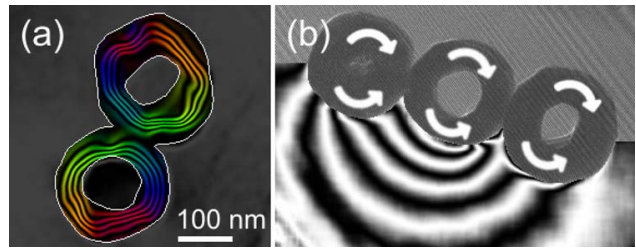

FIG. 2. (Color online) (a) Magnetic induction in two magnetite rings after magnetization. The opposite circulation direction of the vortex state in both rings is the result of the parallel magnetization in the connecting region to minimize the energy. (b) Off-axis electron hologram and magnetic induction of three magnetite rings. The magnetization inside (visualized by the arrows) leads to a stray field outside the rings similar to a dipole.

the chain. The coupling leads to the formation of an extended onion state. ${ }^{10,12}$ Here, the magnetization follows the perimeters of the rings, entering and leaving the rings at opposite ends of the chain which leads to a large stray field with a dipole character, as seen in Fig. 2(b).

Finally, we look at what happens when the ring thickness is increased in order to study the transition when going to a tubelike structure. A very thick magnetite ring (ring 3: average diameter of $185 \mathrm{~nm}$, average width of $60 \mathrm{~nm}$, thickness up to $135 \mathrm{~nm}$ ) is studied and here we observe a very different magnetization state [Fig. 3(a)]. In contrast to the other rings, we do not observe the vortex state but rather a magnetic state with two vortices with opposite sense of rotation inside the ring itself and several magnetic domains are visible. The two adjacent vortices have an antiparallel circulation giving parallel magnetization in the region where they meet in order to minimize the magnetic energy. To understand the reasons for this different kind of magnetization configuration, we investigate the three-dimensional ring geometry by HAADF electron tomography and obtain a three-dimensional reconstruction of the ring [Fig. 3(b) and electronic physics auxiliary publication service (EPAPS) $\left.{ }^{20}\right]$. As already found in the second sample, there is a strong variation in thickness $(85-135 \mathrm{~nm})$. The comparison of the three-dimensional reconstruction and the magnetic induction map shows that the vortices are at the regions of the largest thickness. At these positions, where $t \gg w$ the energetically favorable direction for the magnetization is out-of-plane in the direction of the longest axis ( $z$ axis) which is along the thickness. The magnetization in the vortex cores is pointing perpendicular to the ring plane ( $z$ direction). In the thinner parts of the ring, the magnetization is in the ring plane. This observation indicates that the magnetization configuration of this third ring is an intermediate state between the in-plane vortex and the tube state where the magnetization is completely aligned parallel to the tube axis ( $z$ direction).

In conclusion, using TEM, we have studied the geometric, crystallographic, and magnetic properties of threedimensional single crystal magnetite rings. Investigating the magnetization configuration of both isolated rings and exchange coupled rings, we have found that the fine details of the geometry and the presence of neighboring rings govern the magnetization configuration. In most rings, the magnetization forms the flux closure vortex state. When thickness variations are present, a stray field results even for the vortex state. As the ring thickness is increased above the ring width, the magnetization starts to point out of plane. Using direct
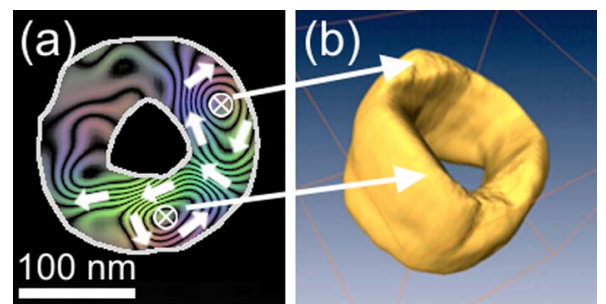

FIG. 3. (Color online) (a) After magnetization, several vortices and domains are visible in the magnetic induction visualized by the small arrows. (b) HAADF tomographic reconstruction of a single ring (see Ref. 20). The two long arrows indicate the two regions with the largest ring thickness corresponding to the position of the two vortices with opposite sense of rotation seen in (b).

high resolution tomography, we have shown that the threedimensional ring geometry is directly correlated with the magnetization configuration and for a ring with varying thickness, an intermediate magnetic state is found with the magnetization pointing in-plane in the thin parts and forming vortices going out of plane in the thick parts of the ring.

The authors acknowledge support by the Deutsche Forschungsgemeinschaft (SFB 513), the Landesstiftung Baden-Württemberg, and the EPSRC(UK). M. E. acknowledges support by the DAAD and R. E. D.-B. acknowledges support by the Royal Society.

${ }^{1}$ J.-G. Zhu, Y. Zheng, and G. A. Prinz, J. Appl. Phys. 87, 6668 (2000).

${ }^{2}$ M. M. Miller, G. A. Prinz, S.-F. Cheng, and S. Bounnak, Appl. Phys. Lett. 81, 2211 (2002).

${ }^{3}$ Yu. S. Dedkov, U. Rüdiger, and G. Güntherodt, Phys. Rev. B 65, 064417 (2002).

${ }^{4}$ J. L. Tsai, K. T. Huang, W. C. Cheng, M. D. Lee, T. S. Chin, S. U. Jen, S. F. Lee, and Y. D. Yao, J. Magn. Magn. Mater. 272, 1664 (2004).

${ }^{5}$ A. Bollero, M. Ziese, R. Höhne, H. C. Semmelhack, U. Köhler, A. Setzer, and P. Esquinazi, J. Magn. Magn. Mater. 285, 279 (2005).

${ }^{6}$ J. J. Versluijs and J. M. D. Coey, J. Magn. Magn. Mater. 226, 688 (2001). ${ }^{7}$ M. Ziese and H. J. Blythe, J. Phys.: Condens. Matter 12, 1 (2000).

${ }^{8}$ M. Kläui and C. A. F. Vaz, in Handbook of Magnetism and Advanced Magnetic Materials, edited by H. Kronmüller and S. S. P. Parkin (Wiley, Chichester, 2007), Vol. 2.

${ }^{9}$ A. Hubert and R. Schäfer, Magnetic Domains-The Analysis of Magnetic Microstructures (Springer, Berlin, Heidelberg, New York, 1998).

${ }^{10}$ J. Rothman, M. Kläui, L. Lopez-Diaz, C. A. F. Vaz, A. Beloch, J. A. C. Bland, Z. Cui, and R. Speaks, Phys. Rev. Lett. 86, 1098 (2001).

${ }^{11}$ Y. Zheng and J.-G. Zhu, J. Appl. Phys. 81, 5471 (1997).

${ }^{12}$ M. Kläui, C. A. F. Vaz, L. Lopez-Diaz, and J. A. C. Bland, J. Phys.: Condens. Matter 15, R985 (2003).

${ }^{13}$ M. Kläui, C. A. F. Vaz, J. A. C. Bland, W. Wernsdorfer, G. Faini, and E. Cambril, Appl. Phys. Lett. 81, 108 (2002).

${ }^{14}$ M. Laufenberg, D. Bedau, H. Ehrke, M. Kläui, U. Rüdiger, D. Backes, L. J. Heyderman, F. Nolting, C. A. F. Vaz, J. A. C. Bland, T. Kasama, R. E. Dunin-Borkowski, S. Cherifi, A. Locatelli, and S. Heun, Appl. Phys. Lett. 88, 212510 (2006).

${ }^{15}$ C. J. Jia, L. D. Sun, Z. G. Yan, L. P. You, M. Takano, N. Hayashi, K. Zheng, X. D. Han, Z. Zhang, and C.-H. Yan (unpublished).

${ }^{16}$ P. A. Midgley and M. Weyland, Ultramicroscopy 96, 413 (2003).

${ }^{17}$ R. E. Dunin-Borkowski, M. R. McCartney, and D. J. Smith, Encyclopaedia of Nanoscience and Nanotechnology, edited by H. S. Nalwa (American Scientific, Los Angeles, California, 2004), Vol. 3, pp. 41-100.

${ }^{18}$ R. J. Harrison, R. E. Dunin-Borkowski, and A. Putnis, Proc. Natl. Acad. Sci. U.S.A. 99, 16556 (2002).

${ }^{19}$ M. Kläui, C. A. F. Vaz, and J. A. C. Bland, Appl. Phys. Lett. 86, 032504 (2005).

${ }^{20}$ See EPAPS Document No. E-APPLAB-92-074821 for an animated video of the high-angle annular dark-field (HAADF) tomographic reconstruction of a single magnetite ring. For more information on EPAPS, see http:// www.aip.org/pubservs/epaps.html. 